\title{
ON INTENSITIES OF MODULATED COX MEASURES
}

\author{
JEWGENI H. DSHALALOW \\ Department of Applied Mathematics \\ Florida Institute of Technology \\ Melbourne, FL 32901, U.S.A. \\ e-maileugene@winnie.fit.edu
}

(Received April, 1997; Revised March, 1998)

\begin{abstract}
In this paper we introduce and study functionals of the intensities of random measures modulated by a stochastic process $\xi$, which occur in applications to stochastic models and telecommunications. Modulation of a random measure by $\xi$ is specified for marked Cox measures. Particular cases of modulation by $\xi$ as semi-Markov and semiregenerative processes enabled us to obtain explicit formulas for the named intensities. Examples in queueing (systems with state dependent parameters, Little's and Campbell's formulas) demonstrate the use of the results.

Key words: Markov modulated Poisson process, modulated Cox measure, Little's formula, Campbell's formula, potential for modulation, semi-Markov modulated Cox process, semiregenerative modulated Cox process.
\end{abstract}

AMS subject classifications: Primary $60 \mathrm{G} 57,60 \mathrm{~K} 15,60 \mathrm{~K} 99$, secondary $60 \mathrm{~K} 10,60 \mathrm{~K} 25$.

\section{Introduction}

This work is inspired by the popularity of Markov modulated Poisson processes (MMPP's), which widely occur in telecommunications [1,10,17] and queueing [14], in particular, in queues with state dependent parameters $[7,15]$. The basic notion of an MMPP is as follows. Let $\xi$ be a Markov chain with continuous time parameter and finitely many states: $\left\{s_{1}, \ldots, s_{m}\right\}$. Suppose we have $m$ (component) Poisson processes, $\Pi_{1}, \ldots, \Pi_{m}$ with intensities $\lambda_{1}, \ldots, \lambda_{m}$, respectively. We will merge them in one process, say $\Pi$, as follows. While $\xi$ is in state $s_{k}, \Pi=\Pi_{k}$. As soon as $\xi$ changes its state to $s_{j}, I$ assumes $\Pi_{j}$ and so on. Obviously, $\Pi$ can also be described in terms of a Cox process with stochastic intensity being a function of $\xi$. Such processes are common in telecommunications, where $m$ input signals at a node are governed by a relay switching to one of the $m$ positions. This relay is contorolled itself by a Markov chain. The output signal is moving on to a next node where it is further processed. In various applications, such a signal is an arrival process in a queueing system. These queueing models were developed by Neuts and his followers [9,11-13]. Rather than 
ordinary arrivals, Neuts [11] further allowed bulk arrivals referred to as batch Markov modulated Poisson processes (BMMPP's).

Whereas Neuts generalized BMMPP's by augmenting multiple exponential phases, all governed by the Markov chain $\xi$ (a so-called versatile Markov process), our research takes a completely different direction - modifying the BMMPP and targeting the intensity of the $\xi$-modulated process. Here we allow $\xi$ to be more general than a Markov chain with finitely many states, and we upgrade each component Poisson processes to a marked Cox process. Various applications to queueing, such as Little's formula, $L=\lambda W$ or its generalization, such as $H=\lambda G$ and Campbell's formula (where $\lambda$ stands for such intensity) [16,18], and optimization [7], motivated the author to study these problems in $[5,6,8]$.

In this article, the author refines the concepts and results initiated in $[5,6,8]$ and further extends them to a class of functionals of the intensity. The paper is organized as follows. Section 2 provides a minimal background on random measures and BMMPP's and introduces to the notation used throughout the paper. Section 3 deals with the formalism of Cox measures modulated by a random process $\xi$ and evaluates the potential for $\xi$-modulation. Section 4 contains the main results of the paper. It introduces functional intensities of modulated Cox measures and provides a background to special cases of $\xi$ treated in Section 5 . Section 5 deals with $\xi$ as semiMarkov and semi-regenerative processes. Here the author obtains explicit formulas for the intensities of Cox measures modulated by such processes. The latter find applications to the prominent queueing formulas $L=\lambda W$ and $H=\lambda G$, which, among other examples in queueing, are demonstrated in Section 6.

\section{Random Measures}

The following are basic notions of random measures outlined in this section for consistency and to comply with the notation throughout the paper. For more information, the reader is referred to monograph [4] by Daley and Vere-Jones.

Let $E$ be a Polish space. Denote by $\mathfrak{M}$ the set of all Radon measures on the Borel $\sigma$-algebra $\mathfrak{B}(E)$. Recall that a measure $\mu$ is Radon if $\mu$ is finite on the ring $\mathscr{R}$ of all relatively compact subsets of $E$. For instance, the Lebesgue measure on $\mathscr{B}\left(\mathbb{R}^{n}\right)$ is an example of a Radon measure.

A Radon measure is called a point or counting measure if $\mu(R) \in \mathbb{N}_{0}$ for all $R \in \Re$. A point measure is simple if for each $x \in E, \mu(\{x\})$ is either 0 or 1 . A Radon measure $\mu_{a}$ is purely atomic if there is a nonempty countable set $\left\{a_{1}, a_{2}, \ldots\right\} \subseteq E$ and a sequence $\left\{\alpha_{1} \geq 0, \alpha_{2} \geq 0, \ldots\right\}$ with $0<\sum_{n \geq 1} \alpha_{n}<\infty$ such that

$$
\mu_{a}=\sum_{n=1}^{\infty} \alpha_{n} \varepsilon_{a_{n}}
$$

where $\varepsilon_{a}$ is the Dirac point mass. The Radon measure $\mu_{d}$ is diffuse if $\mu_{d}(\{x\})=0$ for all $x \in E$. Any Radon measure can be decomposed as

$$
\mu=\mu_{a}+\mu_{d}
$$

$\mathfrak{M}_{p}, \mathfrak{M}_{a}$, and $\mathfrak{M}_{d}$ stand for the sets of all point, atomic, and diffuse measures, respectively.

Let $(\Omega, \mathcal{F})$ be a measure space. A random measure is a measurable mapping from 
$(\Omega, \mathscr{F})$ to $(\mathfrak{M}, \mathscr{B}(\mathfrak{M}))$. In other words, a random measure is, on one hand, a family of Radon measures indexed by $\omega \in \Omega$; on the other hand, it is a family of random variables (r.v.'s) indexed by all Borel sets. A random measure is point, atomic, or diffuse, if in the above definition, $\mathfrak{M}$ is replaced by $\mathfrak{M}_{p}, \mathfrak{M}_{a}$, or $\mathfrak{M}_{d}$, respectively.

Let $\tau=\left\{\tau_{1}, \tau_{2}, \ldots\right\}$ be a sequence of r.v.'s valued in $E$. Then, the random measure

$$
M=\sum_{j \geq 1} n_{j} \varepsilon_{\tau_{j}}, \text { with } n_{j} \in \mathbb{N}_{0}
$$

is an element of $\mathfrak{M}_{p} . M$ is purely atomic if all $\tau$ 's are discrete, and diffuse if all $\tau$ 's are continuous r.v.'s. Given $M$ above,

is called its support counting measure.

$$
N=\sum_{j \geq 1} \varepsilon_{\tau_{j}}
$$

For $\nu \in \mathfrak{M}_{d}$, the random measure $\pi_{\nu}=\sum_{j \geq 0} e^{-\nu} \frac{\nu^{n}}{n !} \varepsilon_{n}$ is called a Poisson measure directed by a (diffuse) measure $\nu$. A Poisson measure can also be directed by a (diffuse) random measure $\Lambda$, in notation, $\pi_{\Lambda}$.

A point random measure $\Pi$ is called a Poisson (counting) process directed by a measure $\nu \in \mathfrak{M}_{d}$, if for each Borel set $A, \Pi(A)$ is a Poisson process with mean $\nu(A)$, or, equivalently,

$$
\mathbb{E}\left[z^{\Pi(A)}\right]=e^{\nu(A)(z-1)} .
$$

$\nu$ is called the mean measure of $\Pi$.

A point random measure $C$ is called a Cox measure or Cox process or doubly stochastic Poisson process directed by a random measure $\Lambda$ if

1) given $\Lambda$ and disjoint Borel sets $A_{1}, \ldots, A_{n}$, the r.v.'s $C\left(A_{1}\right), \ldots, C\left(A_{n}\right)$ are independent,

2) $P_{C}=\pi_{\Lambda}$ or, equivalently, $\mathbb{E}\left[z^{C(A)} \mid \Lambda\right]=e^{\Lambda(A)(z-1)}$.

Let $E=\mathbb{R}^{m}$ and $\mathcal{L}$ denote the Lebesgue measure on $\mathscr{B}\left(\mathbb{R}_{+}^{m}\right)$. Suppose the mean measure $\nu$ (of a Poisson process $\Pi$ ) is absolutely continuous and let $\lambda \in \frac{d \nu}{d \mathscr{L}}$ be a version of its Radon-Nikodym derivative. We assume that $\nu$ is such that $\lambda$ is strictly positive. Such $\lambda$ is called the rate or intensity function of the Poisson process $\Pi$. In particular, if $\lambda$ is constant, the Poisson process $\Pi$ is called homogeneous or stationary. If $C$ is a Cox process directed by a random measure $\Lambda$ then the corresponding rate function $\lambda$ is called the stochastic intensity. In other words, $\lambda$ is a stochastic process and it is related with $\Lambda$ by

$$
\Lambda(\cdot, A)=\int_{A} \lambda(\cdot, x) \mathcal{L}(d x)
$$

In applications, the stochastic intensity is a function of a stochastic process $\xi: \lambda$ $=h(\xi)$. In particular, if $\xi$ is a Markov process, then $C$ is an MMPP.

Let $X=\left\{X_{0}, X_{1}, \ldots\right\}$ be a sequence of $\mathbb{R}_{+}{ }^{\text {-valued r.v.'s. The random measure }}$

$$
M=\sum_{j \geq 1} X_{j} \varepsilon_{\tau_{j}}
$$

is called a marked Poisson process if its support counting measure $\Pi=\sum_{j \geq 1} \varepsilon_{\tau_{j}}$ is Poisson. If $I$ is a Cox process then $M$ is a marked Cox process. We will denote a marked Poisson process by the triple $\langle M, \nu, X\rangle$, and a marked Cox process by $\langle M, \Lambda, X\rangle$. If the mean measure $\Lambda$ is expressed through a stochastic intensity $\lambda$ 
$=\lambda(\xi), \xi$ is a Markov process, and $X_{i}$ 's depend upon $\xi$, then such a marked Cox process will be denoted $M^{\xi}=\left\langle M^{\xi}, \lambda(\xi), X^{\xi}>\right.$ and referred to as the BMMPP.

\section{Modulated Cox Measures}

In the above definition of the BMMPP it is assumed that $\xi$ is a jump Markov process with finitely many states, say $\left\{s_{1}, \ldots, s_{m}\right\}$, so that if $\xi$ enters state $s_{k}$, the intensity of the input becomes $\lambda_{k}=\lambda\left(s_{k}\right)$ and the respective marks (arriving batches) will assume index $k$, i.e. $X^{(k)}=\left\{X_{1}^{(k)}, X_{2}^{(k)}, \ldots\right\}$. This status of the input remains unchanged while $\xi$ stays in $s_{k}$. As soon as $\xi$ enters another state the arrival Poisson process alters accordingly.

Below we offer an alternative and more explicit construction of such a process. Let $M=\left\{M_{k}, k=1, \ldots, m\right\}$ be an $m$-tuple of marked homogeneous Poisson processes

$$
M_{k}=\sum_{j \geq 1} X_{j}^{(k)} \varepsilon_{\tau_{j}^{(k)}}
$$

with intensity $\lambda_{k}$. Then, the BMMPP $M^{\xi}$ can be represented by

$$
M^{\xi}(\omega, A)=\sum_{k=1}^{m} M_{k}\left(\omega, A \cap S_{k}(\omega)\right)
$$

where $S_{k}=\xi^{-1}\left(\left\{s_{k}\right\}\right)$. This construction is also advantageous for the formation of the intensity of $M^{\xi}$. Before we turn to the intensities we would like to extend the notion of a modulated process by allowing $\xi$ to be an arbitrary separable stochastic process. If the state space of $\xi$ is countable then the above construction (3.2) is the same, except for $m$ being replaced by $\infty$. Should the state space of $\xi$ be more than countable, we would like to reduce it to a countable decomposition for the convenience of the next construction.

Let $\left\{\Omega, \Im,\left(P^{j}\right)_{j \in \Psi}, \xi(\cdot, t), t \in E\right\} \rightarrow\{\Psi, \mathscr{B}(\Psi)\}$ be a separable stochastic process. For $H \in \mathscr{B}(\Psi), S=\{(\omega, t) \in \Omega \times T: \xi(\omega, t) \in H\}$ is the sojourn time of $\xi$ in $H$ on a time set $T$. [We will assume that $T$ is relatively compact.] Therefore, $S=\xi^{-1}(H) \cap(\Omega \times T)$ is a random set. For instance, if $\langle M, \Lambda, X\rangle$ is a marked Cox process, in which the discrete-valued mark $X_{i}$ gives the size of the $i$ th arrival batch at $\tau_{i}$, then

$$
M(S)=\sum_{j \geq 1} X_{j} \varepsilon_{\tau_{j}}(S)
$$

gives the number of arrivals of $M$ during the sojourn time of the process $\xi$ in $H$ on a time set $T$.

Let $\mathcal{H}_{6}=\left\{H_{0}, H_{1}, \ldots\right\}$ be a sequence of subsets of $\Psi$ such that $\bigcup_{n=0}^{\infty} H_{n}$ is a measurable decomposition of $\Psi$, called $\mathcal{H}$-decomposition of $\Psi$, and let $M=\left\{M_{n} ; n=0,1, \ldots\right\}$ be a sequence of random measures. If $S_{n}$ denotes the sojourn time of $\xi$ in $H_{n}$ on the time set $T$, then obviously, $\bigcup_{n=0}^{\infty} S_{n}$ is a measurable decomposition of the cylinder set $\Omega \times T$. Let $\mathfrak{M}_{T}$ denote the set of all Radon measures on the trace Borel $\sigma$-algebra $\mathscr{B}(E) \cap T$. Then, the "decomposition" of $M$ with respect to $\xi$ and $\mathfrak{H}$, 


$$
M^{\xi}(\omega, A)=\sum_{j \geq 0} M_{j}\left(\cdot, \xi^{-1}\left(H_{j}\right) \cap T\right)
$$

is obviously a random measure from $(\Omega, \mathscr{F})$ to $\left(\mathfrak{M}_{T}, \mathfrak{B}\left(\mathfrak{M}_{T}\right)\right)$. The following is a refined definition of a modulated random measure originally introduced in Dshalalow [5].

Definition 3.1. We will call $M^{\xi}$ in (3.3) the random measure modulated by $\xi$ (on $T$ ) given $\mathcal{H}$-decomposition and the sequence (of random measures) $M=\left\{M_{0}, M_{1}, \ldots\right\}$, or, alternatively, the $\xi$-modulation of $M$ given $\mathcal{H}_{6}$. In particular, if the components $M_{j}=\left\langle M_{j}, \Lambda_{j}, X^{(j)}>, j=0,1, \ldots\right.$, are Cox measures $M^{\xi}$ will be referred to as a $\xi$ modulated Cox measure given $\mathcal{H}$-decomposition and Cox measures $M=$ $\left\{<M_{j}, \Lambda_{j}, X^{(j)}>; j=0,1, \ldots\right\}$, or a $\xi$-modulated Cox measure, for short. If $M$ $=\left\{\left\langle M_{j}, \nu_{j}, X^{(j)}\right\rangle ; j=0,1, \ldots,\right\}$ are marked Poisson measures, then $M^{\xi}$ will be called a $\xi$-modulated marked Poisson process.

When the process $\xi$ enters the set $H_{k}, M^{\xi}$ turns to $M_{k}$ and remains equal $M_{k}$ as long as $\xi$ stays in $H_{k}$, i.e. during its sojourn time in set $H_{k}$, in our case, traced on a relatively compact time set $T$. Thus, given $\xi \in H_{k}, M^{\xi}$ is a marked Cox process with stochastic intensity $(\omega, x) \mapsto \lambda_{k}(\omega, x)$ and marks $X^{(k)}=\left(X_{r}^{(k)} ; r=1,2, \ldots\right)$. In the special case, when $\lambda_{k}$ are constants and $\xi$ is a jump Markov process with finitely many states, $M^{\xi}$ reduces to the BMMPP.

Definition 3.2. Given a sequence $M=\left\{M_{0}, M_{1}, \ldots\right\}$ of random measures, denote

$$
\mathcal{M}^{\xi}(T)=\mathcal{M}^{\xi}(\cdot, T)=\sum_{j \geq 0} M_{j}\left(\cdot, S_{j}\right)=\sum_{j \geq 0} M_{j}\left(\cdot, \xi^{-1}\left(H_{j}\right) \cap T\right)
$$

and call $\mathbb{E}\left[\mathcal{H}^{\xi}(T)\right]$ the $M$-potential for $\xi$-modulation given $\mathfrak{J}$.

For example, if the marks $X$ are discrete-valued, the random variable $\mathcal{H}^{\xi}(T)$ can be interpreted as the total number of arrivals on a time set $T$. Note that if we let $T$ vary, then $\mathcal{H}^{\xi}$ in $(3.4)$ becomes a random measure from $(\Omega, \mathscr{F})$ to $(\mathfrak{M}, \mathfrak{B}(\mathfrak{M}))$. We can also assume that each $M_{k}$ is a Cox measure directed by a random measure $\Lambda_{k}$ if another dependence is required.

Definition 3.3 Let $T$ be a relatively compact set in $E$ and let $\mu \in \mathfrak{M}$. If $\mu(T) \neq 0$ we define

$$
\kappa_{T}=\frac{\mathbb{E}\left[\mathcal{H}^{\xi}(T)\right]}{\mu(T)}=\frac{\mathbb{E}\left[\sum_{j \geq 0} M_{j}\left(S_{j}\right)\right]}{\mu(T)}
$$

and call it the intensity of the modulated measure $M^{\xi}$ on set $T$ with respect to measure $\mu$. Suppose there exists a monotone increasing family $\left\{T_{\alpha} ; \alpha \in \mathcal{N}\right\}$ of relatively compact subsets of $E$ along a net $\mathcal{N}$ such that $T_{\alpha} \uparrow E$. If the limit

$$
\kappa=\lim _{\alpha \in \mathcal{N}}\left(\kappa_{T}\right)
$$

exists we call it the intensity of $M^{\xi}$ with respect to measure $\mu$.

Now we make the following assumptions. For each $j \in \mathbb{N}_{0}$, the marks $X^{(j)}$ $=\left\{X_{r}^{(j)} ; r \in \mathbb{N}\right\}$ represent a stationary sequence of r.v.'s, with common mean $\alpha_{j}$, independent of their positions $\tau^{(j)}=\left\{\tau_{r}^{(j)} ; r \in \mathbb{N}\right\}$. Thus, given $\Pi_{j}$, for each relatively compact set $R$, 


$$
M_{j}(R)=M_{j}(\cdot, R)=\sum_{n=1}^{\infty} X_{n}^{(j)} \varepsilon_{\tau_{n}^{(j)}}(R)=\sum_{\left\{n: \tau_{r}^{(j)} \in R\right\}} X_{n}^{(j)}, \text { a.s. }
$$

where $\Pi_{j}$ is the associated support counting measure directed by a Radon measure $\nu_{j}$. Then, (3.7) yields

$$
\mathbb{E}\left[\mathbb{E}\left[M_{j}(R) \mid \Pi_{j}\right]\right]=\alpha_{j} \mathbb{E}\left[\Pi_{j}(R)\right]=\alpha_{j}{ }_{j}(R) .
$$

If $R$ is a random set, independent of $X$ 's,

(3.8) is modified as

$$
\mathbb{E}\left[\mathbb{E}\left[\mathbb{E}\left[M_{j}(R) \mid \Pi_{j}\right] \mid R\right]\right]=\alpha_{j} \mathbb{E}\left[\nu_{j}(R)\right] .
$$

Let $R=S_{j}=\xi^{-1}\left(H_{j}\right) \cap T$ (for each $\omega \in \Omega$ ). Obviously

$$
\nu_{j}\left(S_{j}\right)=\int_{T} I_{H_{j}}(\xi) d \nu_{j}
$$

and, if $\nu_{j}$ is absolutely continuous, then due to (2.1) and the above assumptions imposed on the intensities $\lambda_{j}$ 's (as being constants on $S_{j}$ ), (3.10) yields that

$$
\nu_{j}\left(S_{j}\right)=\lambda_{j} \int_{T} I_{H_{j}}(\xi) d \mathcal{L} .
$$

In a more general setting, where $\lambda_{j}$ varies on $S_{j}$, (3.10) leads to

$$
\nu_{j}\left(S_{j}\right)=\int_{T} I_{H_{j}}(\xi(x)) \lambda_{j}(x) \mathcal{L}(d x) .
$$

Finally, if the measure, directing $\Pi_{j}$, is random (in notation $\Lambda_{j}$ ) then (3.12) turns to

$$
\Lambda_{j}\left(S_{j}\right)=\int_{T} I_{H_{j}}(\xi(x)) \lambda_{j}(\cdot, x) \mathcal{L}(d x),
$$

where $\lambda_{j}$ is the stochastic intensity of $\Pi_{j}$. Now, from (3.4), (3.8), and (3.11), along with the monotone convergence theorem, we have that

$$
\mathbb{E}\left[\mathcal{M}^{\xi}(T)\right]=\sum_{j \geq 0} \alpha_{j} \lambda_{j} \int_{T} \mathbb{P}\left\{\xi \in H_{j}\right\} d \mathcal{L} .
$$

If measure $\Lambda_{j}$, directing $\Pi_{j}$, is random, then (3.9), under $(*)$, should be modified as

$$
\mathbb{E}\left[\mathbb{E}\left[\mathbb{E}\left[\mathbb{E}\left[M_{j}(R) \mid \Pi_{j}\right] \mid R\right] \mid \Lambda_{j}\right]\right]=\alpha_{j} \mathbb{E}\left[\mathbb{E}\left[\Pi_{j}(R) \mid R, \Lambda_{j}\right]\right]
$$

and in light of (3.13) it leads to

$$
\mathbb{E}\left[\mathcal{H}^{\xi}(T)\right]=\sum_{j \geq 0} \alpha_{j} \mathbb{E}\left[\int_{T} I_{H_{j}}(\xi(x)) \lambda_{j}(\cdot, x) \mathcal{L}(d x)\right]
$$

If, given $\xi^{-1}\left(H_{j}\right), \xi$ and $\lambda_{j}$ are uncorrelated, then from (3.16), by Fubini's theorem we have that

$$
\mathbb{E}\left[\mathcal{H}^{\xi}(T)\right]=\sum_{j \geq 0} \alpha_{j} \int_{T} \mathbb{P}\left\{\xi(x) \in H_{j}\right\} \lambda_{j}(x) \mathcal{L}(d x),
$$

where $\lambda_{j}(x)=\mathbb{E}\left[\lambda_{j}(\cdot, x)\right]$. In particular, if for each $j, M_{j}$ is a stationary Cox 
measure, i.e., $\lambda_{j}(\cdot, x)=\lambda_{j}(\cdot)$, then (3.17) yields the same formula as (3.14) for $\mathbb{E}\left[\mathcal{M}^{\xi}(T)\right]:$

where $\lambda_{j}=\mathbb{E}\left[\lambda_{j}(\cdot)\right]$.

$$
\mathbb{E}\left[\mathcal{M}^{\xi}(T)\right]=\sum_{j \geq 0} \alpha_{j} \lambda_{j} \int_{T} \mathbb{P}\left\{\xi \in H_{j}\right\} d \mathcal{L}
$$

Note that (3.14) and (3.16-3.18) hold true also for real-valued $\alpha_{j}$. The above results can be summarized as the following theorem.

Theorem 3.1. Let $M^{\xi}$ be a $\xi$-modulated Cox measure (on T) given J6decomposition with respect to Cox measures $M=\left\{\left\langle M_{j}, \Lambda_{j}, X^{(j)}\right\rangle ; j=0,1, \ldots\right\}$. Then, the $M$-potential for $\xi$-modulation satisfies formula (3.16) with the conditions imposed on $X^{(j)}$ and $\Lambda_{j}$ :

marks $X^{(j)}$ represent stationary sequences, with common means

$\alpha_{j}$, independent of their positions $\tau^{(j)}$,

$\Lambda_{j}$ have stochastic intensities $\lambda_{j}$.

If, given $\xi^{-1}\left(H_{j}\right), \xi$ and $\lambda_{j}$ are uncorrelated, the M-potential reduces to formula (3.17), where $\lambda_{j}(x)=\mathbb{E}\left[\lambda_{j}(\cdot, x)\right]$. If, in addition, $M_{j}$ are stationary Cox measures (with $\lambda_{j}=\mathbb{E}\left[\lambda_{j}(\cdot)\right]$ ) then $M$-potential further reduces to formula (3.18). [The same result as in (3.18) holds true for nonstochastic intensities $\lambda_{j}$.]

\section{Functional Rates of Modulated Cox Measures}

The following will generalize the above formulas (3.14) and (3.16-3.18) for the case of reward functions applied to $\xi$ on respective sojourn times. The idea is to replace indicator functions $I_{H_{j}}$ in (3.11-3.13) by arbitrary Borel functions $\varphi_{j}$.

\section{Definition 4.1.} us call

(i) Assume that $\varphi=\left\{\varphi_{0}, \varphi_{1}, \ldots\right\}$ is a sequence of Borel measurable functions. Let

$$
M^{\varphi(\xi)}=\sum_{j \geq 0} M_{j} \varphi_{j}(\xi)
$$

the $\xi$-modulation of $M$ given $\varphi$, which we also denote by $<M^{\varphi(\xi)}, M=\left\{M_{j}\right.$ $\left.=\sum_{i \geq 1} X_{i}^{(j)} \varepsilon_{\tau_{i}^{(j)}} ; j=0,1, \ldots\right\}, \varphi=\left(\varphi_{0}, \varphi_{1}, \ldots\right)>$. [For $\varphi \geq 0, M^{\varphi(\xi)}$ is a random measure $(\Omega, \mathfrak{F})$ to $(\mathfrak{M}, \mathfrak{B}(\mathfrak{M}))$.]

(ii) Given a relatively compact subset $T$ of $E$, denote

$$
\mathcal{M}^{\varphi(\xi)}(T)=\sum_{j \geq 0} \int_{T} \varphi_{j}(\xi) d M_{j}
$$

and call the value $\mathbb{E}\left[\mathcal{H}^{\varphi(\xi)}(T)\right]$ the $M$-potential for $\xi$-modulation given $\varphi$.

For instance, if $M_{j}=\sum_{i \geq 1} X_{i}^{(j)} \varepsilon_{\tau_{i}^{(j)}}$, where $X_{i}^{(j)}$ stands for the size of the $i$ th batch of type $j$ and the state space of $\xi$ is discrete, and $\varphi_{j}(x)=c_{j}(x) I_{\{j\}}(x)$, then $\int_{T} \varphi_{j}(\xi) d M_{j}$ gives the reward for all arrivals on time set $T$ of type $j$ during the sojourn time of $\xi$ in state $\{j\}$. Thus, the $M$-potential gives the total reward of all 
arrivals during the time set $T$ subject to $\xi$.

Lemma 4.2. Let $M=\sum_{j \geq 0} X_{j} \varepsilon_{\tau_{j}}$ be a marked Cox measure directed by a random measure $\Lambda$ and led by a stationary sequence $X=\left\{X_{1}, X_{2}, \ldots\right\}$ of marks with common mean $\alpha<\infty$, independent of their positions $\tau=\left\{\tau_{1}, \tau_{2}, \ldots\right\}$. Then, for a Borel measurable function $\varphi$, the stochastic integral $\alpha \int \varphi(\xi) d \Lambda$ is a version of the conditional expectation $\mathbb{E}\left[\int \varphi(\xi) d M \mid \Lambda\right]$.

Proof. It is sufficient to show the validity of the Lemma for $\varphi \geq 0$, since to turn to the general case, we will apply it for $\varphi^{+}$and $\varphi^{-}$in the decomposition $\varphi=\varphi^{+}-\varphi^{-}$.

Given $\varphi \geq 0$, there is a sequence $\left\{s_{n}\right\}$ of simple functions with $\sup \left\{s_{n}\right\}=\varphi$. Let

$$
s_{n}=\sum_{r=1}^{l_{n}} a_{r}^{(n)} I_{A_{r}^{(n)}}{ }^{.}
$$

Denote $h(\Lambda)=\mathbb{E}\left[\int \varphi(\xi) d M \mid \Lambda\right]$. By change of variables formula,

$$
\begin{gathered}
h(\Lambda)=\mathbb{E}\left[\int \varphi d M \xi^{-1} \mid \Lambda\right]=\mathbb{E}\left[\sup \sum_{r=1}^{l_{n}} a_{r}^{(n)} M \xi^{-1}\left(A_{r}^{(n)}\right) \mid \Lambda\right] \\
=\sup \sum_{r=1}^{l_{n}} a_{r}^{(n)} \mathbb{E}\left[M \xi^{-1}\left(A_{r}^{(n)}\right) \mid \Lambda\right] .
\end{gathered}
$$

Let $I$ be the support counting measure for $M$. Then, because of (3.7),

$$
\begin{gathered}
\mathbb{E}\left[M \xi^{-1}\left(A_{r}^{(n)}\right) \mid \Lambda\right]=\mathbb{E}\left[\mathbb{E}\left[M \xi^{-1}\left(A_{r}^{(n)}\right) \mid \Pi\right] \mid \Lambda\right] \\
=\alpha \mathbb{E}\left[\Pi \xi^{-1}\left(A_{r}^{(n)}\right) \mid \Lambda\right]=\alpha \Lambda \xi^{-1}\left(A_{r}^{(n)}\right) .
\end{gathered}
$$

The latter yields that

$$
h(\Lambda)=\alpha \sup \int s_{n} d \Lambda \xi^{-1}=\alpha \int \varphi d \Lambda \xi^{-1}
$$

The following theorem gives an explicit expression for the $M$-potential, $\mathbb{E}\left[M^{\varphi(\xi)}\right]$, generalizing the results of Theorem 3.1.

Theorem 4.3. Let $M=\left\{\left\langle M_{j}, \Lambda_{j}, X^{(j)}\right\rangle, j=0,1, \ldots\right\}$ be a sequence of marked Cox measures. Then, the $M$-potential for $\xi$-modulation given $\varphi$ satisfies the following formula:

$$
\mathbb{E}\left[\mathcal{M}^{\varphi(\xi)}(T)\right]=\mathbb{E}\left[\sum_{j \geq 0} \int_{T} \varphi_{j}(\xi) d M_{j}\right]=\sum_{j \geq 0} \alpha_{j} \mathbb{E}\left[\int_{T} \varphi_{j}(\xi) d \Lambda_{j}\right]
$$

Proof. The statement follows from Lemma 4.2 and the monotone convergence theorem.

Corollary 4.4. In the conditions of Theorem 4.3, let $E=\mathbb{R}^{m}, \Lambda_{j}$ be absolutely continuous for each $\omega$ and let $\lambda_{j}$ be a version of the corresponding Radon-Nikodym derivative. Assume further that, given $\Lambda_{j}, \lambda_{j}$ is independent of $\xi, j=0,1, \ldots$ Then, the $M$-potential for $\xi$-modulation satisfies the following formula:

$$
\mathbb{E}\left[\mathcal{H}^{\varphi(\xi)}(T)\right]=\sum_{j \geq 0} \alpha_{j} \int_{T} \mathbb{E}\left[\varphi_{j}(\xi(x))\right] \lambda_{j}(x) \mathcal{L}(d x)
$$


where $\lambda_{j}(x)=\mathbb{E}\left[\lambda_{j}(\cdot, x)\right]$. In particular, if $M_{j}$ is a stationary marked Cox measure, $j=0,1, \ldots$, then $(4.4)$ reduces to

$$
\mathbb{E}\left[\mathcal{H}^{\varphi(\xi)}(T)\right]=\sum_{j \geq 0} \alpha_{j} \lambda_{j} \int_{T} \mathbb{E}\left[\varphi_{j}(\xi(x))\right] \mathcal{L}(d x),
$$

where $\lambda_{j}=\mathbb{E}\left[\lambda_{j}(\cdot)\right]$.

Proof. The corollary follows directly from (4.3) by applying Fubini's theorem and using the conditional expectation with respect to $\Lambda_{j}$.

Formula (4.5) further reduces to (3.18) for $\varphi_{j}=I_{H_{j}}$.

In (3.5), we defined the intensity of the modulated random measure $M^{\xi}$ on $T$ with respect to a Radon measure $\mu$ as

and in (3.6) by

$$
\begin{aligned}
& \kappa_{T}=\frac{\mathbb{E}\left[\mathcal{M}^{\xi}(T)\right]}{\mu(T)} \\
& \kappa=\lim _{\alpha \in \mathcal{N}}\left(\kappa_{T}\right)
\end{aligned}
$$

the intensity of $M^{\xi}$. Now, for $I_{T} M^{\varphi(\xi)}$, we define the function $T \mapsto \mathbb{E}\left[I_{T} \mathcal{M}^{\varphi(\xi)}\right]$ on a set $T$ as

and and analogously,

$$
\kappa_{T}(\varphi)=\frac{\mathbb{E}\left[\mathcal{M}^{\varphi(\xi)}(T)\right]}{\mu(T)}
$$

$$
\kappa(\varphi)=\lim _{\alpha \in \mathcal{N}} \kappa_{T}(\varphi)
$$

calling them, the reward rate on set $T$ and reward rate (with respect to $\mu$ ), respectively.

\section{Reward Rates and Intensities for Special Processes}

This section will deal with the evaluation of reward rates and intensities for $\xi$ as semi-Markov and semiregenerative processes. Throughout this section we make the following assumption.

Assumption 1. $E=\mathbb{R}_{+}$and $\mu$ is the Lebesgue measure. $<M^{\varphi(\xi)}, M=\left\{M_{j}\right.$ $\left.=\sum_{i \geq 1} X_{i}^{(j)} \varepsilon_{\tau_{i}^{(j)}} ; j=0,1, \ldots\right\}, \varphi=\left\{\varphi_{0}, \varphi_{1}, \ldots\right\}>$ is the $M$-modulation for $\xi$, where $\varphi_{j}: \Psi \rightarrow \mathbb{R}$. [See Definition 4.1.] $M_{j}$ 's are stationary marked Cox processes with stochastic intensities $\lambda_{j}(\cdot), \lambda_{j}=\mathbb{E}\left[\lambda_{j}(\cdot)\right]$, led by stationary sequences $X^{(j)}$ of marks with common finite means $\alpha_{j}$.

Notation. For a vector $x$ with entries indexed by elements of $\Psi$ and a Borel-measurable function $g$ defined on $\Psi$, denote $x g=\sum_{r \in \Psi} x_{r} g(r)$. In all other instances $x y$ denotes the usual scalar product. The vector $x \circ y$ stands for the Hadamard (entrywise) product of vectors $x$ and $y . \lambda=\left\{\lambda_{j} ; j \in \Psi\right\}, \alpha=\left\{\alpha_{j} ; j \in \Psi\right\}$, and $\Phi$ denotes the matrix with row elements $\left\{\varphi_{j}(k), k \in \Psi\right\}, j \geq 0$. Define the transformation $\Gamma: \mathbb{R}^{\infty} \rightarrow \mathbb{R}$ as 


$$
\Gamma(x)=(\alpha \circ \lambda)^{\mathrm{T}}(\Phi x) .
$$

\section{$5.1 \xi$ as a Semi-Markov Process}

Let $\zeta=\sum_{i=0}^{\infty} \xi_{i} \varepsilon_{T}$, be an irreducible and aperiodic Markov renewal process with a discrete state space $\Psi$ for $\xi_{n}$. Denote $\beta_{j}=\mathbb{E}^{j}\left[T_{1}\right]$ and $\beta=\left(\beta_{j} ; j \in \Psi\right)$. Suppose that the embedded Markov chain $\left\{\xi_{n}\right\}$ is ergodic and that $p=\left(p_{j} ; j \in \Psi\right)$ is its invariant probability measure. We call the Markov renewal process $\zeta$ recurrent-positive if its mean inter-renewal time, denoted as scalar product $p \beta$, is finite. An irreducible, aperiodic, and recurrent-positive Markov renewal process is called ergodic. Let $N=\sum_{j \geq 0} \varepsilon_{T_{j}}$ denote the support counting measure associated with $\zeta$. Then, $\xi(x)=\zeta_{N([0, x])}$ is known to define the semi-Markov process associated with the Markov renewal process $\zeta$. The semi-Markov process $\xi$ is ergodic if so is $\zeta$. We assume that the Markov renewal process $\zeta$ is ergodic.

For some Borel set $B$ let

and

$$
\Theta^{j}(j, B)=\mathbb{E}^{j}\left[\int_{B} g(\xi) d \ell\right]
$$

$$
\boldsymbol{\Theta}^{j}(B)=\left\{\Theta^{j}(j, B) ; j \in \Psi\right\}
$$

where $g$ is a Borel-measurable function with compact support defined on $\Psi$.

Proposition 5.1. (cf. Cinlar [3]). Let $\xi$ be the semi-Markov process associated with an ergodic Markov renewal process $\zeta$ and let $p$ be the invariant probability measure of the embedded Markov chain $\xi_{n}$. Then for some Borel function $g$ on $\Psi$ and for all $j \in \Psi$, the limit

$$
\lim _{t \rightarrow \infty} \frac{1}{t} \Theta^{j}([0, t])=\frac{(p \circ \beta)^{\mathrm{T}} g}{p \beta}
$$

exists and it is independent of $j$.

Now, using Proposition 5.1 (for $\varphi_{j}$ replacing $g$ ) and formula (4.6) from Corollary 4.4 for $T=[0, t]$, we establish:

Theorem 5.2. Under Assumption 1, the $M$-potential for $\xi$-modulation is determined from the formula:

$$
\kappa(\varphi)=\lim _{t \rightarrow \infty} \frac{1}{t} \mathbb{E}\left[\mathcal{H}^{\varphi(\xi)}([0, t])\right]=\frac{1}{p \beta} \Gamma(p \circ \beta) .
$$

\section{$5.2 \xi$ as a Semiregenerative Process}

\section{Definition 5.1}

(i) Let $T$ be a stopping time for a stochastic process $\left\{\Omega, \mathcal{F},\left(P^{j}\right)_{j \in \Psi}, \xi(t) ; t \geq 0\right\}$ $\rightarrow(\Psi, \mathfrak{B}(\Psi)) . \xi$ is said to have the locally strong Markov property at $T$, if for each bounded random variable $\zeta: \Omega \rightarrow \Psi^{r}$ and for each Baire function $f: \Psi^{r} \rightarrow \mathbb{R}$, $r=1,2, \ldots$, it holds true that

$$
E^{j}\left[f\left(\zeta \circ \theta_{T}\right) \mid \mathscr{F}_{T}\right]=E^{\xi_{T}}[f(\zeta)] \quad P^{j} \text {-a.s. on }\{T<\infty\}
$$


where $\theta_{y}$ is the shift operator and o stands for the composition.

(ii) A separable stochastic process $\left\{\Omega, \mathcal{F},\left(P^{j}\right)_{j \epsilon \Psi}, \xi(t) ; t \geq 0\right\} \rightarrow(\Psi, \mathfrak{B}(\Psi))$ with $\Psi \preceq \mathbb{N}$ and a.e. right-continuous paths is called semi-regenerative if

a) there is a counting measure $N=\sum_{n=1}^{\infty} \varepsilon_{T_{n}}$ on $\mathbb{R}_{+}$such that $T_{n} \rightarrow \infty$ $(n \rightarrow \infty)$ and such that each $T_{n}$ is a stopping time relative to the filtration $\sigma\left(\xi_{t} ; t \leq T_{n}\right)$

b) the process $\xi$ has the locally strong Markov property at $T_{n}, n=1,2, \ldots$,

c) $Z=\sum_{i=0}^{\infty} \xi_{i} \varepsilon_{T_{i}}, \xi_{n}=\xi\left(T_{n}\right)$, is a Markov renewal process.

$\xi$ is ergodic if so is $Z$.

The following Proposition is similar to one in Çinlar [2].

Proposition 5.3. Let $\xi$ be an ergodic semi-regenerative process with the stationary probability measure $\pi$ and let $g$ be a Borel-measurable function on $\Psi$ with compact support. Then

$$
\lim _{t \rightarrow \infty} \frac{1}{t} \int_{0}^{t} g(\xi) d \mathcal{L}=\pi g
$$

Theorem 5.4. Under Assumption 1, the $M$-potential for $\xi$-modulation is determined from the formula:

$$
\kappa(\varphi)=\lim _{t \rightarrow \infty} \frac{1}{t} \mathbb{E}^{j}\left[\mathcal{M}^{\varphi(\xi)}([0, \mathrm{t}])\right]=\Gamma(\pi) .
$$

Proof. Formula (5.5) follows from Proposition 5.3 (for $\varphi_{j}$ replacing $g$ ) and formula (4.6), from Corollary 4.4, for $T=[0, t]$.

\section{Examples and Applications}

Example 6.1. Consider a class of queueing systems in which the queueing process $Q(t)$ is semiregenerative, for instance, queues of type $M / G / 1$ and $G / M / m$. Let $\zeta=\left\{Q_{n}, T_{n}\right\}$ and $\xi$ be the associated Markov renewal and semi-Markov processes, respectively. Here $\left\{Q_{n}\right\}$ is the embedded Markov chain over the sequence $\left\{T_{n}\right\}$ of arrivals or departures of groups of customers. Let $M^{Q}$ denote the input as $Q$ modulated marked Cox process and let $\varphi_{j}(k)=c_{j}(k) I_{\{j\}}(k)$ be the reward function for mode $k$. Then,

$$
\mathcal{M}^{\varphi(Q)}(T)=\sum_{j \geq 0} \int_{T} \varphi_{j}(Q) d M_{j}
$$

gives the total reward for all arrivals on time set $T$ and $\kappa(\varphi)=\Gamma(\pi)$ is the reward rate of the arrival process (over infinite horizon) modulated by the queueing process $Q$. If in (6.1) we replace $Q$ by $\xi$, we arrive at a "restricted modulation." For instance, if $T_{n}$ is the $n$th departure time then the arrival process gets modulated only at departure instants. Then, the reward rate is $\kappa(\varphi)=\frac{1}{p \beta} \Gamma(p \circ \beta)$. If $T_{n}$ is the $n$th arrival epoch then the arrival process gets modulated by the system correspondingly at arrival instants. All above systems refer to as "queues with state dependent parameters." 
Finally, the arrival process can be modulated by an "external" semi-Markov or semiregenerative process, for instance, by a totally separate stochastic system.

Example 6.2. Let $\varphi_{j}=I_{\{j\}}$. Then, formulas (5.3) and (5.5) will reduce to

and

$$
\kappa=\frac{1}{p \beta} p(\alpha \circ \beta \circ \lambda)
$$

$$
\kappa=\pi(\alpha \circ \lambda)
$$

respectively, earlier obtained in Dshalalow [6]. In a widely referred to Little's formula $L=\lambda W$, where $L$ and $W$ are the mean queue length and the mean sojourn time, respectively, $\lambda$ stands for the intensity of the input process. Replacing $\lambda$ by $\kappa$ in $(6.2)$ and (6.3) we specify Little's formula

$$
L=\kappa W
$$

for a large class of queues and other stochastic systems with modulated input processes. A similar use of $\kappa$ is for generalizations of Little's formula, such as $H=\kappa G$ and Campbell's formula, see Whitt [18] and Schmidt and Serfozo [16].

Example 6.3. Consider the following queueing system of $M / G / 1$ type with state dependent parameters. Customers arrive at a servicing facility in bulk and are successively processed by a single server which waits for customers if it is idle or goes on "vacations." Introduce the following notation:

$\sigma_{n} \sim$ the duration of the $n$th service,

$T_{n} \sim$ the instant of the $n$th departure, $t$ ),

$Q(t) \sim$ the queueing process (number of customers present in the system at time

$$
Q_{n}=Q\left(T_{n}+0\right)
$$

Given $Q_{n}=i$, the probability distribution function of $\sigma_{n+1}$ is $B_{i}$ and on time interval $\left[T_{n}, T_{n+1}\right)$, customers arrive in accordance with the general queuedependent bulk birth process. The latter means that on time interval $\left[T_{n}, T_{n+1}\right)$ the arrival process is a marked Poisson process modulated by $Q(t) . Q(t)$ is a semiregenerative process relative to the Markov renewal process $\left\{Q_{n}, T_{n}\right\}$. A special case of this model, with single arrivals, was studies by Shellhaas [15], who found the stationary distribution, $\pi$, of the queueing process $Q$. Shellhaas' results can further be extended by using above Little's formula (6.4) to arrive at the mean stationary waiting time or to get the reward rate.

\section{Acknowledgement.}

The author is grateful to anonymous referees for their constructive suggestions, which led to an improved presentation of the paper. 


\section{References}

[1] Chen, J.S.-C., Guérin, R., and Stern, T., Markov-modulated flow model for the output queues of a packet switch, IEEE Trans. Comm., 40, No. 6, 1098-110, 1992.

[2] Çinlar, E., Introduction to Stochastic Processes, Prentice-Hall, Englewood Cliffs, NJ 1975.

[3] Çinlar, E., Markov renewal theory, Adv. Appl. Prob., 1, 123-187, 1969.

[4] Daley, D.J. and Vere-Jones, D., An Introduction to the Theory of Point Processes, Springer Verlag, New York, NY 1988.

[5] Dshalalow, J.H., On modulated random measures, JAMSA, 4, No. 4, 305-312, 1991.

Dshalalow, J.H., On applications of Little's formula, JAMSA, 6, No. 3, 271-276,

[6] 1993.

[7] Dshalalow, J.H., First excess level analysis of random processes in a class of stochastic servicing systems with global control, Stoch. Anal. Appl., 12, No.1, 75-101, 1994.

[8] Dshalalow, J.H., Ergodic theorems for modulated stochastic processes, in: Proceedings of the 1st Congress of IFNA at Tampa, Florida, W. de Gruyter, 1745-1755, 1996.

[9] Fischer, W. and Meier-Hellstern, K., The Markov-modulated Poisson process (MMPP) cookbook, Perf. Eval., 18, 149-171, 1992.

[10] Heffes, H. and Lucantoni, D., A Markov modulated characterization of Packetized voice and data traffic and related statistical multiplexer performance, IEEE Journ. Selected Area in Comm., SAC-4, No. 6, 856-868, 1986.

[11] Neuts, M.F., Matrix-Geometric Solutions in Stochastic Models: An Algorithmic Approach, J. Hopkins Univ. Press, Baltimore, MD, 1981.

[12] Neuts, M.F., Structured Stochastic Matrices of $M / G / 1$ Type and Their Applications, Marcel Dekker, New York, 1989.

[13] Neuts, M.F., Matrix-analytic methods in the theory of queues, Chapter 10, 265292, in Advances in Queueing, ed. by J.H. Dshalalow, CRC Press, Boca Raton, FL, 1995.

[14] Prabhu, N.U. and Zhu, Y., Markov-modulated queueing systems, Queueing Sys., 5, 215-246, 1989.

[15] Schellhaas, H., Computation of the state probabilities in $M / G / 1$ queues with state dependent input and state dependent service, OR Spektrum, 5, 223-228, 1983.

[16] Schmidt, V. and Serfozo, R., Campbell's formula and applications to queueing, Chapter 8, 225-242, in Advances in Queueing, ed. by J.H. Dshalalow, CRC Press, Boca Raton, FL 1995.

[17] Stavrakakis, I., Queueing analysis of a class of star-interconnected networks under Markov modulated output process modeling, IEEE Trans. Comm., 40, No. 8, 1345-1357, 1992.

[18] Whitt, W., A review of $L=\lambda W$ and extensions, Queueing Sys., 9, 235-268, 1991. 


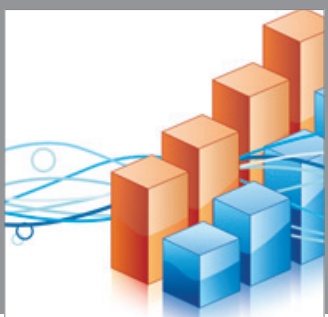

Advances in

Operations Research

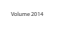

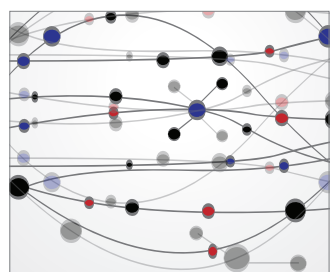

\section{The Scientific} World Journal
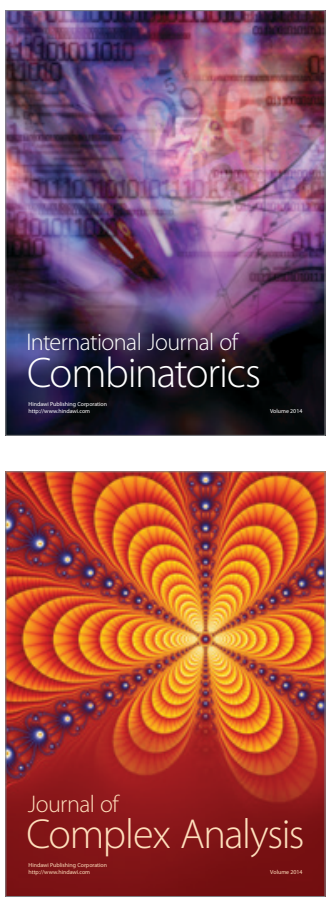

International Journal of

Mathematics and

Mathematical

Sciences
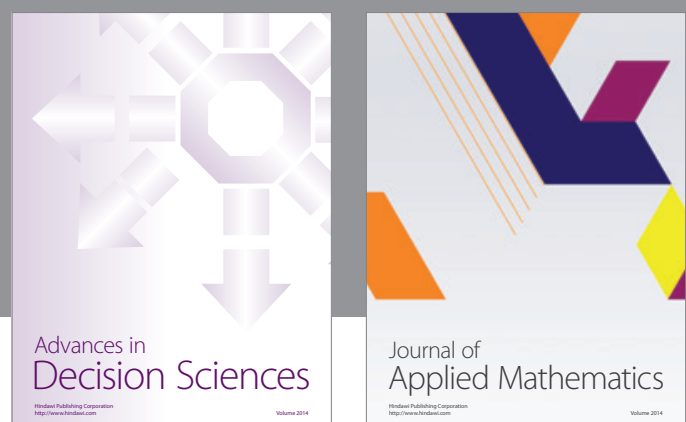

Journal of

Applied Mathematics
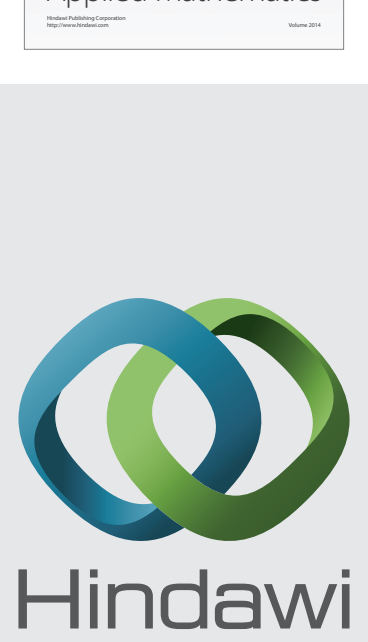

Submit your manuscripts at http://www.hindawi.com
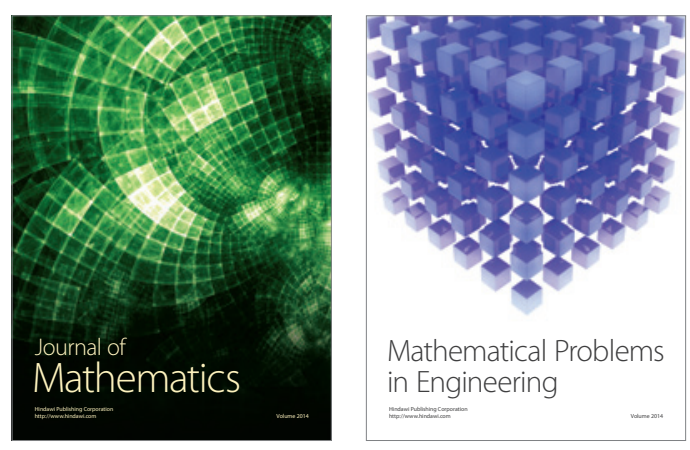

Mathematical Problems in Engineering
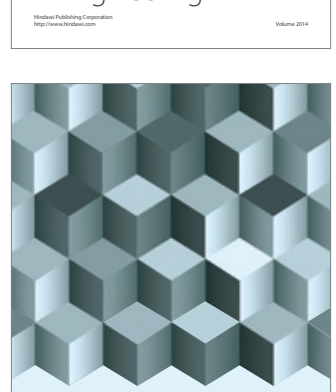

Journal of

Function Spaces
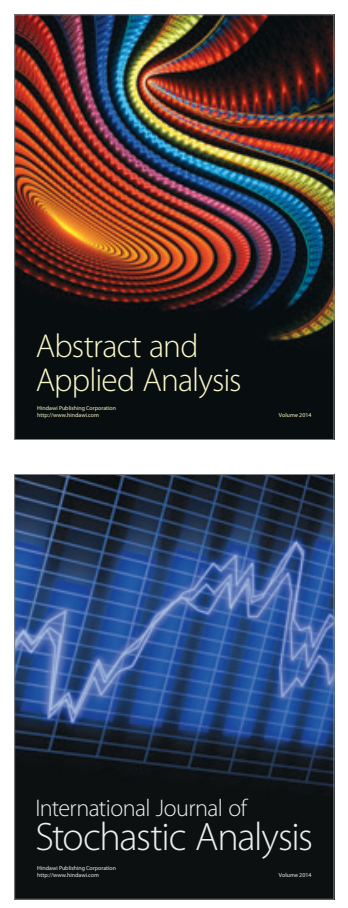

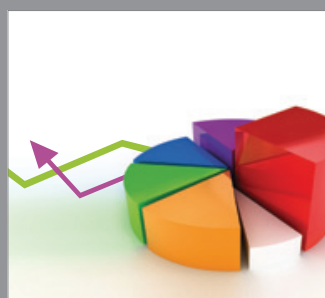

ournal of

Probability and Statistics

Promensencen
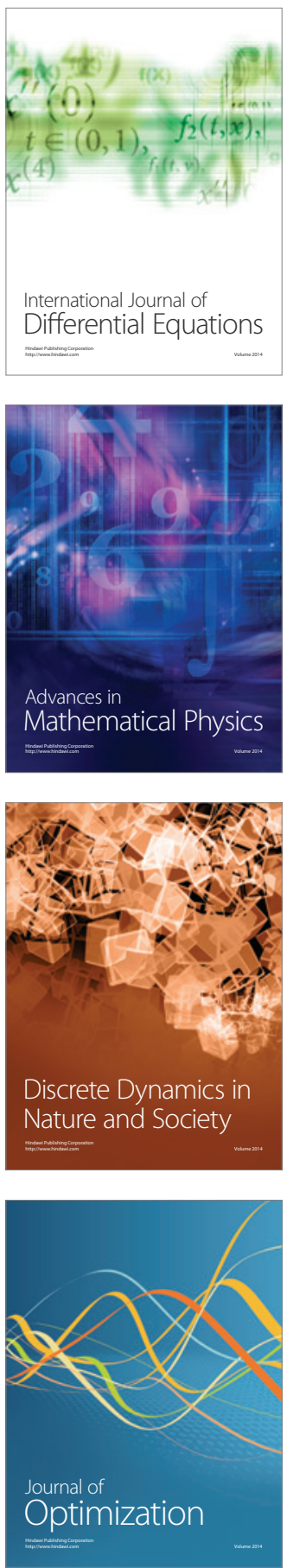